\title{
Mapping Topological Dipole Textures, Chirality, and the Potential Energy Landscape of Polar Skyrmions Using 4D-STEM
}

Yu-Tsun Shao ${ }^{1}$, Sujit Das ${ }^{2}$, Ruijuan Xu ${ }^{3}$, Javier Junquera ${ }^{4}$, Swathi Chandrika ${ }^{1}$, Harold Y. Hwang ${ }^{5}$, Ramamoorthy Ramesh ${ }^{2}$ and David Muller ${ }^{1}$

${ }^{1}$ Cornell University, Ithaca, New York, United States, ${ }^{2}$ University of California-Berkeley, Berkeley, California, United States, ${ }^{3}$ Stanford University, Stanford, California, United States, ${ }^{4}$ Universidad de Cantabria, Santander, Cantabria, Spain, ${ }^{5}$ Stanford University, SLAC National Accelerator Laboratory, Stanford, California, United States,

Emergent topological textures in complex oxides has been the fertile ground for exploring new phases and phenomena, exhibiting exotic functionalities. For example, the recent realization of polar vortices and polar skyrmions in $\left(\mathrm{PbTiO}_{3}\right)_{\mathrm{n}} /\left(\mathrm{SrTiO}_{3}\right)_{\mathrm{n}}$ superlattices suggests a perfect system for exploring interesting physical responses, including emergent toroidal order and local negative capacitance [1, 2]. Here, we explore the topologically non-trivial dipole textures of polar skyrmions, where local variations of ferroelectric polarization, chirality, electric field, and potential energy landscape are resolved using scanning electron nanodiffraction and a high-speed electron microscope pixel array detector (EMPAD) for collecting full CBED patterns [3].

$\mathrm{A}\left[\left(\mathrm{PbTiO}_{3}\right)_{16} /\left(\mathrm{SrTiO}_{3}\right)_{16}\right] 8$ superlattice is studied here, which the film was fully lifted-off as $2 \mathrm{D}$-like membranes [4] for plan view geometry shown in Fig. 1a. The high dynamic range of the EMPAD allows us to record full CBED patterns without saturation or nonlinearities, enabling simultaneous recording of the transmitted beam and Kikuchi bands. We employed Kikuchi bands (Fig. 1 b \& c) as a more robust means to extract polarity information in the polar skyrmions, as slight crystal mistilts are expected and often dominate the contrast in low-order Bragg reflections [5]. Results of electron diffraction-imaging show that the in-plane polarization curling direction is preserved counter-clockwise (CCW) at room temperature (Fig. $1 \mathrm{~d} \& \mathrm{f}$ ). When heated to $373 \mathrm{~K}$, an ordered skyrmion square lattice appeared in which a CCW curling dipole moment was surrounded by arrays of anti-vortices and clockwise curling dipole moments (Fig. 1 e \& g).

To determine the chiralities of the polar order parameter field, 3D information can be accessed by observing the intensity variations in high-order Laue zone (HOLZ) reflections [6, 7]. We deliberately tilted $\sim 6.2^{\circ}$ off-zone along one of the mirror planes to excite the HOLZ reflections (Fig. 2a). Figure 2 (b) shows a virtual dark field image of the ordered skyrmions reconstructed from the SCBED dataset using (771) reflection. By comparing experimental with simulated intensities of HOLZ reflections (line profiles drawn from yellow box in Fig. 2a), we can determine the chiralities of neighboring skyrmions with alternating handedness. Furthermore, the sum of a periodic array of skyrmions and anti-vortices yields zero net chirality (blue curve; Fig. 2c), in accordance with the prediction of Poincaré-Hopf theorem [8, 9].

To map the potential energy landscape, both ferroelectric polarization and electric field need to be measured independently and simultaneously. For this study, we reconstruct the long-range electric fields by measuring the deflection of (000) beam as there are fewer polarity effects. For the cross-section (xzplane) geometry, we can observe the variations in out-of-plane components of polarization and electric field $(\mathrm{Pz} \& \mathrm{Ez})$. Now that both electric and polarization fields are measured, we can thus calculate the local potential energy of the system by integration $G=\int\left(E_{x} \partial_{x} P_{x}+E_{z} \partial_{z} P_{x}\right) d x+\int\left(E_{x} \partial_{x} P_{z}+E_{z} \partial_{z} P_{z}\right) d z$ 
Based on the analysis, the potential energy has local maxima at the domain walls, indicating local regions with negative permittivity [10].

In summary, SCBED in combination with a fast, high dynamic range EMPAD detector allows quantitative imaging polarization, electric field, chirality, and potential energy landscape of complex topological textures such as skyrmions in oxide heterostructures [11].
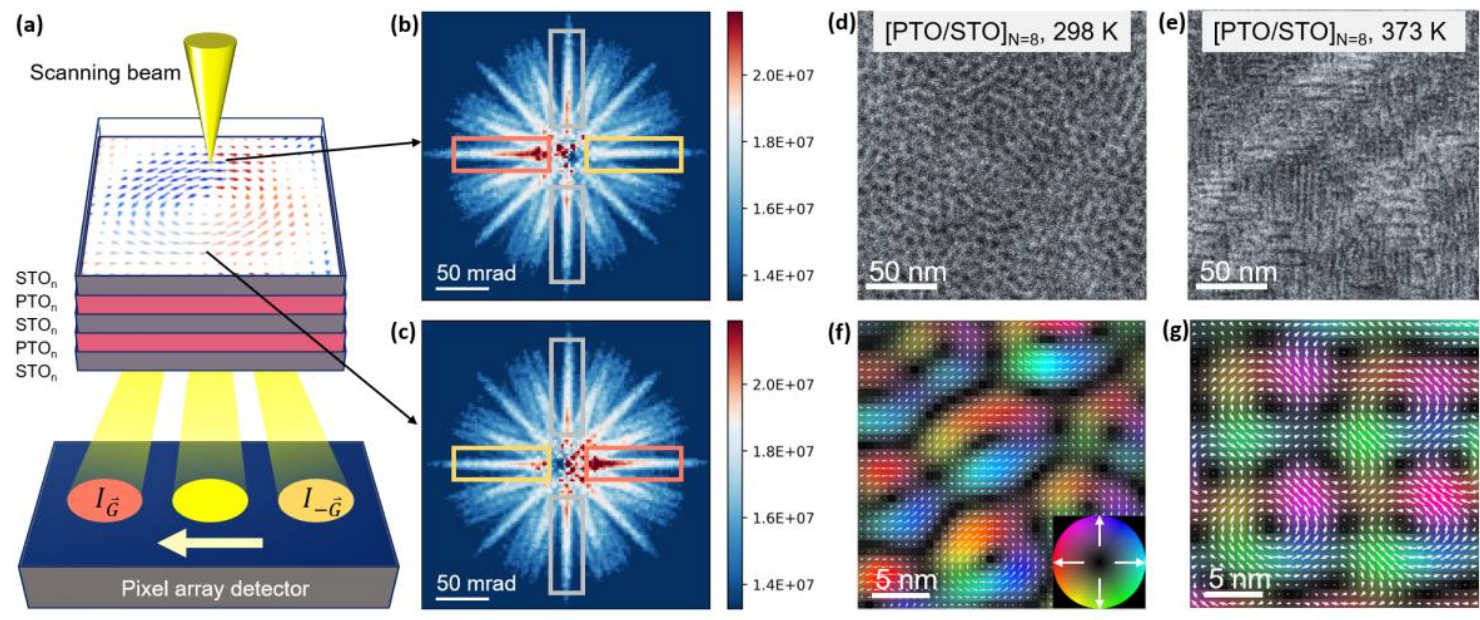

Figure 1. Imaging internal Bloch components of polar skyrmions in the $[(\mathrm{PbTiO} 3) 16 /(\mathrm{SrTiO} 3) 16] 8$ superlattice. (a) Schematic of the SCBED imaging using a scanning electron probe and pixelated array detector EMPAD, where a diffraction pattern was recorded at each probe position. Ferroelectric polarization direction can be determined by observing the difference of diffracted intensities of Friedel pairs, IG and I-G. Representative CBED patterns taken from (b) top and (c) bottom of a skyrmion, where the polarity-sensitive Kikuchi bands were marked by pink and yellow boxes along the polar directions. For clarity of display, the Kikuchi patterns shown in (c \& e) were weighted by k2, where k denotes the scattering vector from the transmitted spot.
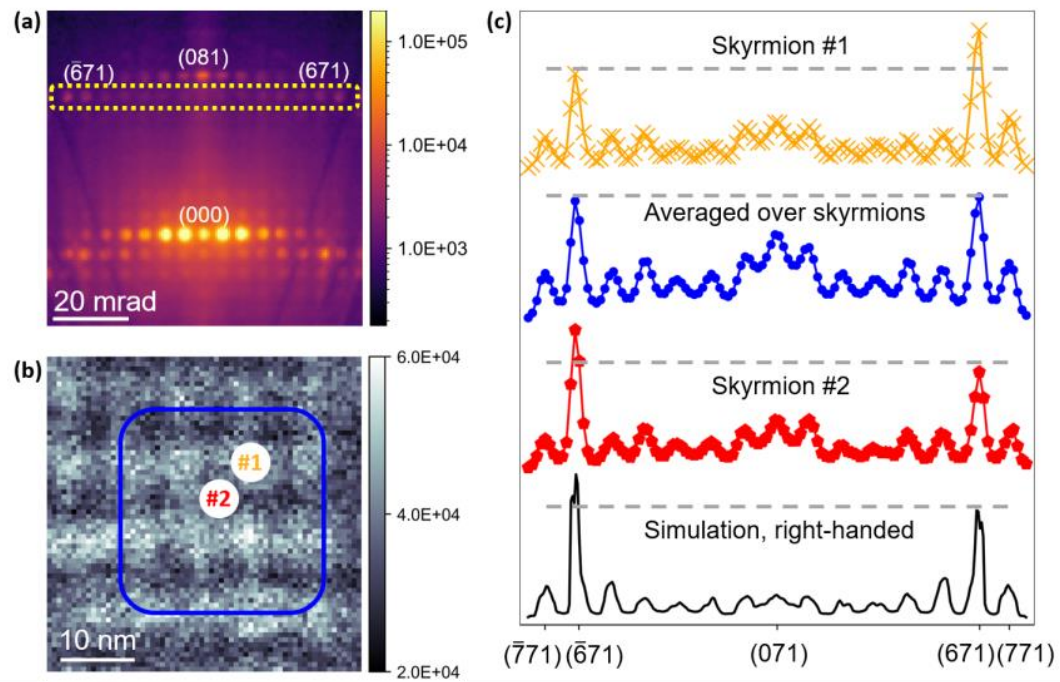

Figure 2. Handedness determination of the chiral polar skyrmions using SCBED. (a) Representative experimental diffraction pattern acquired at an incidence of $\sim 6.2^{\circ}$ away from [001] zone axis, tilted along one of the mirror planes. (b) Virtual dark field image reconstructed from the SCBED dataset using (771) reflection. (c) Intensity profiles of HOLZ reflections from selected regions labeled in (b), displaying the 
intensity difference between two pairs of reflections: (671) and (-671), (771) and (-771). The colored labels in (b) indicates the region which line profiles were drawn from. Colored lines with markers show experimental data, whereas the simulation of a right-handed skyrmion is shown in black line at the bottom.

\section{References}

[1] S. Das, et al., Nature 568 (2019), 368.

[2] A. K. Yadav et al., Nature 565 (2019), 468.

[3] M. W. Tate et al., Microscopy and Microanalysis 22 (2016), 237-249.

[4] D. Lu et al., Nat Mater 15 (2016), 1255.

[5] Y.-T. Shao et al., Microscopy and Microanalysis 25 (Suppl 2), 1938 (2019).

[6] A.W. Johnson, Acta Cryst B63 (2007), 511.

[7] K. Marthinsen, R Høier, EMSA (1989), San Francisco Press, 484.

[8] Y. Nahas. et al., Nat Comm 6 (2015), 8542.

[9] M. A. Pereira Gonçalves et al., Sci Adv 5 (2019), 7023.

[10] S. Das, Y.-T. Shao et al., Nat Mater, in press.

[11] This work is supported by the AFOSR Hybrid Materials MURI, award \# FA9550-18-1-0480. Facilities support from the National Science Foundation (DMR-1429155, DMR-1719875) 